\title{
(Re)inventing Business Networks and Organisations: A Brief Introduction
}

\author{
MISLAV ANTE OMAZIĆ \\ Guest Editor \\ University of Zagreb, Croatia \\ momazic1@efzg.hr \\ https://doi.org/10.26493/1854-4231.12.195-198
}

Management is striving to address and present key issues in international research, first of all from the area of business, economics, governance and management. Largely, this thematic issue is following the MIC 2016 International Conference Managing Global Changes in Pula, Croatia.

The Southeast European economies exhibit common characteristics, but differ greatly at the same time. That is why certain perspectives are applicable although coming outside of the context. The selected papers are written around important but different factors that are affecting modern organisation. Business networks between countries, which once formed one market, have not managed to reinvent themselves. Organizations are instruments, created for the attainment of goals as set up according to organizational vision, based on interest of influential stakeholders. Working toward goals inherently creates change - in activities, structures and resources of the organizations. In this special issue, we use the opportunity to look for answers, which will provide understanding on how organizations in Southeast European countries can improve exchange relationship and successfully reinvent business networks. The performance of the organizations is determined by exchange relationship among organizations in their environments. Further, we seek to understand how organizations may respond and reinvent business environments or how they exert influence upon it. We look for ways to understand management and its mechanisms in their creation of favourable conditions for the creation of knowledge and networks.

In this issue authors from different countries, such as a Croatia, Hong Kong, Turkey Hungary and Slovenia, discuss different themes which are closely related to the (re)invention of business networks 
and organisations by providing research findings which offer theoretical and practical implications for key factors in this scientific field.

The first contribution to the issues is entitled 'Valorisation of Cultural Heritage in Sustainable Tourism,' and it is written by two authors, Kristina Afrić Rakitovac and Nataša Urošević. Modern world is faced with serious shortage of different resources and that is one of many reasons why sustainability became important topic in academic circles. This article reflects upon the models of development of cultural tourism in the city of Pula, Croatia, through crossborder international partnerships. Confronting global trends with local commitment to sustainable development, authors set the hypothesis that heritage tourism, based on the strategic valorisation of unique cultural resources, could strengthen the identity and economy of the local community, create new jobs, increase the quality of life of local residents and the pleasure of visitors, improve the image and attract investors. Authors also provided analysis of different comparable European examples of good practice indicating possible models of sustainable management and valorisation of specific categories of heritage, which could at the same time, enhance the process of urban regeneration and social revitalization. In this article, authors proposed some concrete activities in order to foster development of urban areas and prosperity of their citizens through sustainable tourism.

The second article entitled 'A Validity Study of the MwEP Scale in Hong Kong,' written by authors Tsun-Lok Kwong and Pik-Ching Wan, focuses on the validity of the Multidimensional Work Ethic Profile (MWEP) in the Hong Kong setting. Although, as written, this paper is written within certain context some of its findings are universally applicable. In the modern organisation, issues connected to ethics are neither luxury nor option, it became organizational priority. In this article factor, analysis reveals that similarity exists between factor loadings in original MWEP model and author's model with Hong Kong samples. However, the MWEP items in the current study does not map exactly into the seven dimensions proposed by Miller, Woehr, and Hudspeth in their original work. The leisure and the morality/ethics dimensions match with the original scale respectively with good and poor reliabilities, but the hard work and wasted time dimensions have intertwined and appeared to be inseparable.

The subsequent paper entitled 'The Relationship between Managerial Satisfaction and Job Turnover Intention: The Mediating Role of Job Satisfaction,' by Rüveyda Öztürk Başol and Harun Demirkaya, 
is focused on specific theme within human resource management area. Service sector in Turkey is faced with the huge challenge and that is that growth occurs faster than the other, comparable, sectors. The rapid growth of the number of shopping malls has necessitated the measurement of the attitudes of the employees in this sector. This study demonstrated that the gender, marital status and age were not the significant variables regarding job satisfaction, managerial satisfaction and job turnover intention; however, education status and income status were found to be significant variables on job satisfaction and job turnover intention. In addition, job satisfaction fully mediated the relationship between managerial satisfaction and job turnover intention.

The fourth article is focused on issues connected to knowledge transfer. Viktoria Lambert, author of the paper entitled 'Gamification and the Quality of Informal Post-Experiential Learning,' aims to investigate the enablement of gamified learning tools determined by certain attributes of her research. As corporate learning moves out of the traditional space into the hands of learners, the responsibilities of those who provide guidance in this process are changing. The overall prevalence of learning tools creates a need for competent learning leaders who make decisions about the development of the workforce. A questionnaire survey had been conducted on a selected sample of high-level learning leaders from different countries to examine if there are significant correlations between the usage of gamified learning tools and corporate learning leaders' minds, their competences and the organizational culture profiles of companies. Results had been analysed with the spss statistical software package and indicated that relationships between these variables cannot be categorically proven, so no predictions can be made about the future of gamified learning based on these attributes. It is the highest time to conduct an extensive research to examine relationships between the quality of informal learning and learning tools in the Iст domain.

Finally, Ana Arzenšek and Katarina Košmrlj wrote paper entitled 'Assessment of Relationship between Young Researchers and Mentors and Implications for Knowledge Transfer,' where they observed knowledge transfer and mentorship process in the academic environment. In their paper authors tried to address key factors that are shaping relationships among Slovenian postgraduate students with a 'Young researcher' status and their mentors. They assumed that relationship determines assessment and among other things, they discovered usefulness of the training and knowledge transfer. The 
relevant issues between mentors and mentorees were explored both, quantitatively with a relevant survey and qualitatively with focus groups method. Among other things authors concluded that mentors who encourage the transfer of knowledge are exceptional in their ability to communicate and are teamwork-oriented; they foster active involvement of the candidate in the research group.

We are quite confident that articles in this thematic issue of Management will help our readers to improve their understanding of the science content we covered and challenge them to dig deeper since quest for understand complex matter never ends. We firmly believe that by reading these papers you will recognize many helpful ideas that are going to be valuable impulse for your further research and behaviour. 